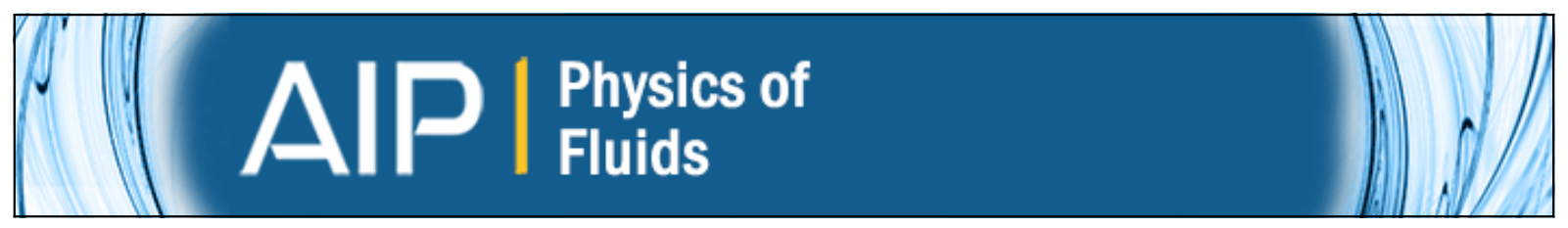

\title{
Vortex synchronization regions in shedding from an oscillating cylinder
}

Fernando L. Ponta and Hassan Aref

Citation: Physics of Fluids (1994-present) 17, 011703 (2005); doi: 10.1063/1.1827275

View online: http://dx.doi.org/10.1063/1.1827275

View Table of Contents: http://scitation.aip.org/content/aip/journal/pof2/17/1 ?ver=pdfcov

Published by the AIP Publishing

\section{Articles you may be interested in}

The vortex shedding around four circular cylinders in an in-line square configuration

Phys. Fluids 26, 024112 (2014); 10.1063/1.4866593

Low-frequency unsteadiness in the vortex formation region of a circular cylinder

Phys. Fluids 25, 085109 (2013); 10.1063/1.4818641

On the lock-on of vortex shedding to oscillatory actuation around a circular cylinder

Phys. Fluids 25, 013601 (2013); 10.1063/1.4772977

The three-dimensional wake of a cylinder undergoing a combination of translational and rotational oscillation in a quiescent fluid

Phys. Fluids 21, 064101 (2009); 10.1063/1.3147935

Vortex formation from a cylinder in shallow water

Phys. Fluids 14, 2957 (2002); 10.1063/1.1483307

\section{AlP Re-register for Table of Content Alerts}

\section{Create a profile.




\title{
Vortex synchronization regions in shedding from an oscillating cylinder
}

\author{
Fernando L. Ponta ${ }^{\text {a) }}$ \\ Department of Theoretical and Applied Mechanics, University of Illinois, Urbana, Illinois 61801 \\ Hassan Aref ${ }^{\text {b) }}$ \\ Department of Engineering Science and Mechanics, Virginia Polytechnic Institute and State University, \\ Blacksburg, Virginia 24061
}

(Received 6 July 2004; accepted 12 October 2004; published online 23 November 2004)

\begin{abstract}
A classification of the vortex patterns in the wake of a normally oscillating cylinder was given by Williamson and Roshko who also studied when the various vortex patterns would occur. Using a symbolic code of letters and numbers that describes the combination of pairs and single vortices shed during each cycle of the forced oscillation of the cylinder, they gave a "map" of vortex-synchronization regions with the wavelength and amplitude of the oscillation as coordinates. In this study, we provide a theoretical basis for the experimental Williamson-Roshko map of vortex synchronization. We show that the region boundaries consist of two families of curves given by specific relations between the values of two nondimensional parameters defining the map. We construct a synthetic map that compares favorably with the experimental data. (C) 2005 American Institute of Physics. [DOI: 10.1063/1.1827275]
\end{abstract}

There is a large body of experimental work on vortex wakes behind cylinders oscillating either normally to or inline with a uniform stream (see Ref. 1, among others). In addition to the experimental studies, there is a more recent body of work utilizing numerical simulations and some theoretical ideas (see Ref. 2 for further references). An influential study by Williamson and Roshko ${ }^{3}$ (henceforth referred to as WR) attempts to classify the vortex wake patterns of a cylinder oscillating normally to the free stream into "synchronization regions." WR use a symbolic code of letters and numbers to describe the combination of pairs and single vortices shed during each cycle of forced oscillation of the cylinder. They denote the observed vortex wake patterns by symbols such as " $\mathrm{P}+\mathrm{S}$," " $2 \mathrm{~S}$," and " $2 \mathrm{P}$," where " $\mathrm{S}$ " signifies a "singlet" or single vortex, and " $P$ " signifies a pair of vortices of opposite signs. A pattern such as " $\mathrm{P}+\mathrm{S}$," then, is one in which in each oscillation cycle the cylinder appears to shed a pair of vortices of opposite sign and a single vortex. Similarly, "2P" signifies a pattern in which two pairs are shed per oscillation cycle, " $2 \mathrm{~S}$ " a pattern in which two singlets are shed per cycle, and so on. The usual von Kármán vortex street would simply be designated " $2 S$ " in this classification. This code has since been widely used in classifications of the vortex structure of wakes. WR gave a "map" delineating when the various vortex patterns would occur [see Fig. 1(a)]. The WR map of vortex patterns uses as coordinates the nondimensional wavelength and nondimensional amplitude of the oscillations of the cylinder, $\lambda / D$, and $A / D$, where $\lambda$ is the wavelength of the cylinder oscillations, $A$ its amplitude, and $D$ is the cylinder diameter. WR observed that the $(\lambda / D, A / D)$ map is partitioned into "synchronization regions" throughout which a certain shedding pattern pre-

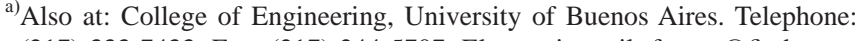
(217) 333-7433. Fax: (217) 244-5707. Electronic mail: fponta@fi.uba.ar

b)Electronic mail: haref@vt.edu
}

vails. Although they studied the case $\mathrm{Re}=392$ in particular detail, and the map in Fig. 1(a) pertains to this specific Reynolds number, they suggest that the regions within the map are invariant over some interval of Reynolds number.

For two opposite vortices shed per cycle, the possible vortex wake patterns are quite limited and are all, essentially, versions of a vortex street with variations in the intervortex spacing. For an oscillating cylinder, more than two vortices can be shed per cycle, and the wake patterns can thus be more complex than the von Kármán street. One may think of the system as a forced, nonlinear oscillator. In this analogy the shedding frequency at a given free stream velocity may be thought of as the natural frequency of the oscillator. The frequency of oscillation of the cylinder is to be regarded as an externally imposed forcing frequency. Due to the nonlinear coupling between these two oscillations a variety of combination frequencies may potentially be excited. Several of these frequencies will correspond to new vortex wake patterns. Even if we restrict ourselves to cases where the wake pattern translates downstream without change of form, i.e., the vortex configuration produced in each oscillation and shedding cycle is identical to that produced in the preceding oscillation/shedding cycle, there are many more possibilities already with three or four vortices per cycle than there are with two.

In this Letter we suggest that the boundaries between the various regions belong to two distinct families, a circumferential family, given by a set of curves concentric with the origin of the map, and a radial family consisting of a second set of curves that emanate radially from the origin. These boundaries are difficult to determine experimentally. Their theoretical basis has hitherto been unclear. We give two heuristic arguments, one relating to the circumferential and the other to the radial boundaries, that produce delineations in the WR map that are surprisingly similar to the experimen- 


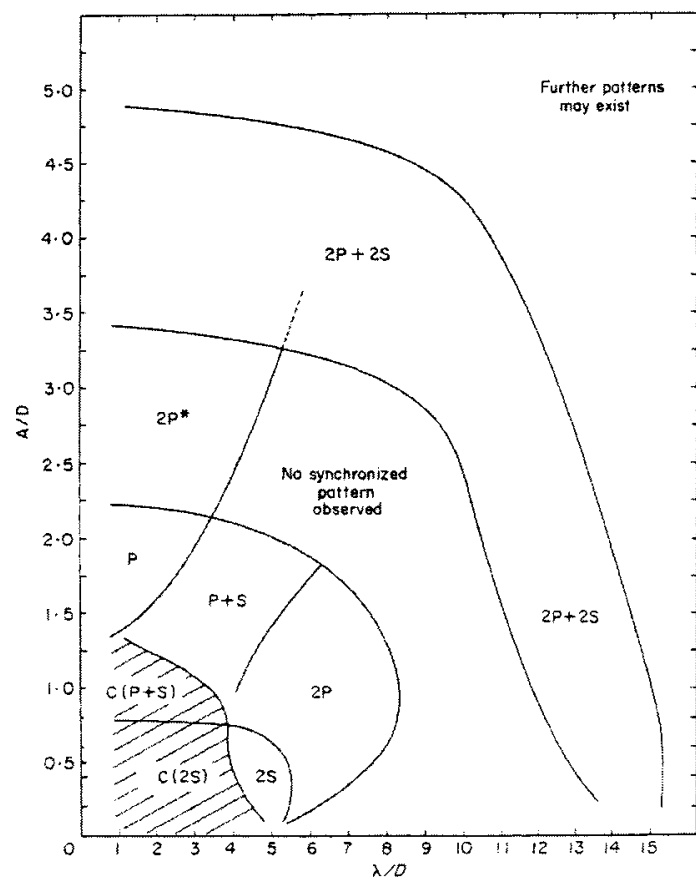

(a)

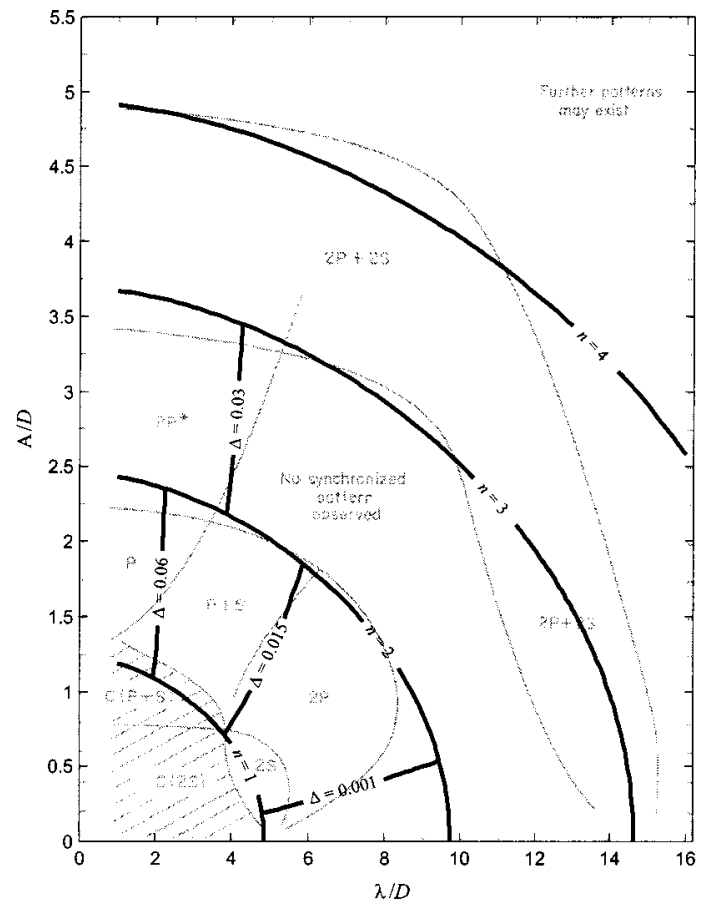

(b)

FIG. 1. (a) Map of vortex synchronization regions in the amplitude-wavelength plane for a normally oscillating cylinder obtained experimentally by Williamson and Roshko (Ref. 3). (b) Synthetic version of the WR map with boundaries defined by the isolines detailed in the text.

tally determined boundaries between vortex synchronization regions.

When a cylinder moves steadily through a fluid at sufficiently high Reynolds number for vortex shedding to occur, it follows from the Navier-Stokes equations that if the shedding takes place in a periodic manner with period $T_{s}$, then the dimensionless Strouhal number, $\mathrm{St}=D /\left(U T_{S}\right)$, must be a universal function of Reynolds number. This relationship is well known experimentally. We have recently provided a theoretical justification for the form of the historically accepted fit to this relation (see Ref. 4). For our present purposes the precise form of the relationship is of less importance than the existence of such a relation in principle. When the cylinder moves through the fluid on an oscillatory path of the form (using an obvious notation)

$$
\begin{aligned}
& x_{c y}(t)=U t, \\
& y_{c y}(t)=A \sin \left(2 \pi \frac{U t}{\lambda}\right),
\end{aligned}
$$

the velocity of the cylinder relative to the fluid is not constant. Our argument, basically, is that both the change in the direction of motion of the cylinder and the change in the speed of its motion affect the shedding pattern of vortices in its wake. As we shall see, the changes in direction are responsible in large measure for the circumferential boundaries in Fig. 1. The changes in speed are responsible for the radial boundaries.

Let us consider the circumferential boundaries first. Figure 2 shows a schematic of the sinuous trajectory of the cylinder. It consists of almost rectilinear segments (marked R in the figure) interrupted by abrupt changes in direction with high curvature (marked $\mathrm{C}$ in the figure) where the angle of inflow changes rapidly.

Now, along the R-sectors of the path we posit that shedding proceeds roughly in the same way as in the case of a steadily translating cylinder. On the other hand, when the cylinder reaches the peaks and troughs of its trajectory (i.e., the $\mathrm{C}$-sectors) the rapid change in the angle of the inflow affects the position of the separation points of the flow on the cylinder and, roughly speaking, interrupts the shedding process, which then recommences along the next following

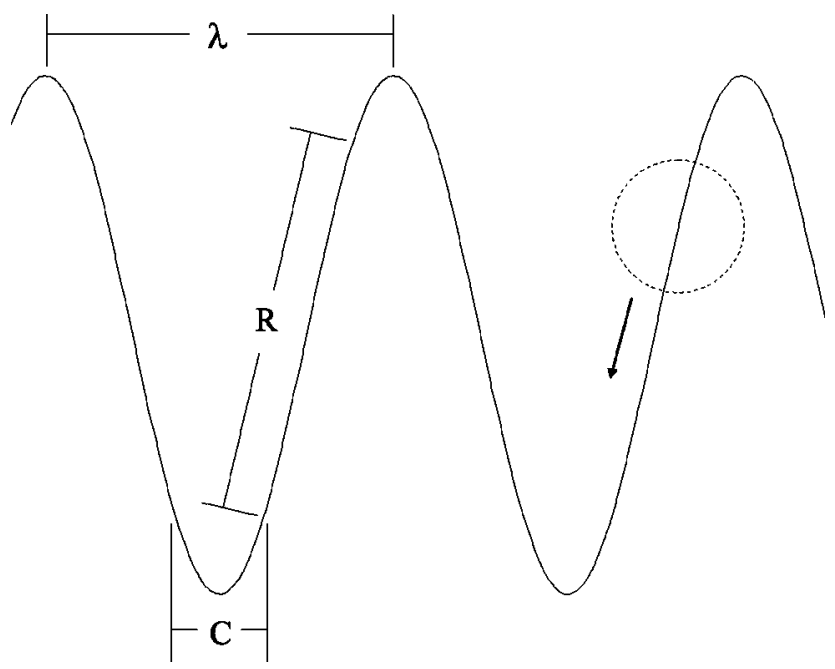

FIG. 2. Schematic view of the sinusoidal trajectory followed by an oscillating cylinder. 
$\mathrm{R}$-sector. Thus, if the length of the R-sector is comparable to the distance traveled by a nonoscillating cylinder during half of a shedding cycle, the oscillating cylinder will shed a pair of vortices of opposite strength during each cycle of its oscillatory motion. If the R-sector length is comparable to twice this distance, there will be two pairs of vortices shed per oscillation cycle, and so on. Our hypothesis is that regions of the amplitude-wavelength map that correspond to trajectories whose R-sector length is comparable to an integer multiple of one half of the distance travelled by a steadily translating cylinder in its natural shedding mode define families of vortex wake patterns that have the same number of vortices shed.

The differences among vortex patterns of the same family (given by the radial boundaries in the WR map) arise from the different processes that the primary vortices undergo after being shed. This hypothesis has some support from experimental observations. Govardhan and Williamson ${ }^{5}$ indicate that a $2 \mathrm{P}$ wake depends on a splitting of both vortices originally shed, due to the straining exerted by neighboring vortices. Thus, instead of having just one vortex pair from each cycle, we produce two pairs. Similarly, we have found in a numerical study (to be published elsewhere) that in the $\mathrm{P}+\mathrm{S}$ wake, vortices of both signs may arise in the near wake, but only one of them splits due to stretching while the other remains strong enough to "recover." In this case we produce three vortices per cycle. The $2 \mathrm{P}$ and $\mathrm{P}+\mathrm{S}$ states, then, depend on similar mechanisms for their generation, namely the splitting of vortices after shedding. Even though the splitting process acts differently for $2 \mathrm{P}$ and $\mathrm{P}+\mathrm{S}$, we start out in both cases with two vortices shed per cycle, one positive and one negative. Hence, both $2 \mathrm{P}$ and $\mathrm{P}+\mathrm{S}$ must belong to the same band in terms of the circumferential boundaries defined by R-sector length, but they are separated by a radial boundary.

To make this argument more quantitative and, in effect, derive an equation for the circumferential boundaries in the WR map, we calculate the length, $L$, of the R-sector for the oscillatory cylinder motion given. This is simply

$$
L=\int_{0}^{T_{e} / 2} \sqrt{U^{2}+\left(2 \pi A \frac{U}{\lambda} \sin \left(2 \pi \frac{U t}{\lambda}\right)\right)^{2}} \mathrm{~d} t,
$$

where $T_{e}=\lambda / U$ is the period of the cylinder oscillation. This may be expressed in terms of the complete elliptical integral of the second kind $E(m)$ as follows:

$$
L=\frac{\lambda}{\pi} E\left(-\left(\frac{2 \pi A}{\lambda}\right)^{2}\right) \text {. }
$$

According to our arguments on shedding mechanisms given above, a circumferential contour in the WR map appears whenever $L$ equals an integer times $U T_{s} / 2$. The equation for these contours then is

$$
\frac{\lambda}{D} \operatorname{St} E\left(-\left(\frac{2 \pi A}{\lambda}\right)^{2}\right)=n \frac{\pi}{2},
$$

where $n$ is an integer and St is the Strouhal number corresponding to the Reynolds number for the free stream. The contours for $n=1,2,3,4$ are shown in Fig. 1(b).
The considerable agreement between the (roughly) circumferential boundaries determined experimentally in the WR map and the theoretical curves is, we believe, significant in spite of the qualitative nature of our argument. The region labeled "No synchronized pattern observed" in the WR map corresponds, in our interpretation, to a situation in which the shedding process is disrupted by the change in the inflow angle (occurring on the C-sectors of the trajectory) before the vortices can be completely shed. Hence, the amount of vorticity contained in the vortices shed during each cycle of the oscillatory motion differs from the circulation of vortices produced during normal shedding from a nonoscillating cylinder. The vortices, then, are unbalanced in size and undergo complex processes of splitting and merging that lead to nonrepetitive patterns. This kind of regime, we suggest, resembles chaotic behavior in the oscillator analogy. In the shaded region close to the origin in Fig. 1(a) WR observed coalescence into a large-scale, von Kármán street-like arrangement of a large number of small vortices produced at high frequencies of the oscillatory motion. This also corresponds to a situation in which the shedding process is interrupted (in this case very frequently) so we have a lot of small vortices shed that, after being advected downstream, merge together to form large von Kármán street-like structures.

A convincing argument for the radial boundaries is more elusive. In terms of our qualitative arguments above, we would have to estimate the rate of strain acting on the newly formed vortices, develop a threshold criterion for whether these split or survive and turn that criterion into a relationship between amplitude and wavelength of the oscillatory cylinder motion. We have not been able to carry through such an argument even approximately. Hence, we have resorted to a more empirical approach in which we have considered the relative change in the period of vortex shedding during the oscillatory motion. If that change exceeds a certain percentage (which we find to be small), changes in the resulting downstream vortex pattern will result. We find that the radial boundaries determined from experiment (to the extent that they really are radial) coincide somewhat with threshold levels of the relative change in shedding period that are integral multiples of 0.005 .

It is important to note that the peak value of the Reynolds number for the flow around the cylinder as it completes its oscillatory trajectory can actually deviate substantially from the free-stream Reynolds number Re. Designating the peak value by a "hat," we have for the trajectory in question

$$
\hat{\operatorname{Re}}=\operatorname{Re} \sqrt{1+\left(2 \pi \frac{A}{\lambda}\right)^{2}} .
$$

The difference between the instantaneous vortex shedding period, $T_{s}$, and its value at peak Reynolds number, $\hat{T}_{s}$, relative to the period of the oscillatory motion, $T_{e}$, is 


$$
\Delta=\frac{T_{s}-\hat{T}_{s}}{T_{e}}=\frac{\frac{D}{U \mathrm{St}}-\frac{D}{U \hat{\mathrm{St}}}}{\frac{\lambda}{U}}=\frac{1 / \mathrm{St}-1 / \hat{\mathrm{St}}}{\lambda / D},
$$

where $\hat{\mathrm{S} t}$ is the Strouhal number associated with $\hat{\mathrm{Re}}$. Taking the $\mathrm{St}-\mathrm{Re}$ relation as summarized by the experimental bestfit line as given by Ref. 6,

$$
S t= \begin{cases}0.212(1-21.1 / \operatorname{Re}), & \operatorname{Re}<180, \\ 0.212(1-12.7 / \operatorname{Re}), & \operatorname{Re}>300,\end{cases}
$$

and substituting this into (5) and then using (4), we may express $\Delta$ in terms of the free-stream Reynolds number, Re, and the WR map coordinates $\lambda / D$ and $A / D$. Other fits (e.g., Ref. 7) to the Re-St relationship could, of course, have been used but the principle that there is such a universal relationship is, once again, more important for our argument than the specific numerical form used.

In Fig. 1(b) we have shown the isolines 0.01, 0.015, 0.03 and 0.06 of $\Delta$ for $\operatorname{Re}=392$. We chose these particular values of $\Delta$ because they appear to roughly coincide with the vortex synchronization region boundaries of the original WR map. We do not understand the origins of the apparent quantization in terms of multiples of 0.005 . We see that a level of relative fluctuation of the shedding period of 0.015 apparently destabilizes the $2 \mathrm{P}$ pattern, which appears to be more sensitive to detuning than $\mathrm{P}+\mathrm{S}$. This is consistent with the idea that the $2 \mathrm{P}$ and $\mathrm{P}+\mathrm{S}$ states depend on similar mechanisms for their generation, but these mechanisms require a finer balance for a $2 \mathrm{P}$ wake to be produced than for a $\mathrm{P}+\mathrm{S}$ wake. As we mentioned above, in both cases a vortex splitting process is key to forming the vortex wake structure, but it is reasonable to assume that the mutual influence of regions of positive and negative vorticity necessary for the $2 \mathrm{P}$ pattern might require a higher degree of synchrony and thus be the more sensitive to fluctuation levels in time scales or frequencies. In our numerical work (to be reported else- where) we have tried moving the cylinder along the same sinusoidal trajectory but at constant speed. We then find that for the range of parameters that for the variable speed trajectory considered here produces the $\mathrm{P}+\mathrm{S}$ wake, the constantspeed counterpart of that trajectory produces a symmetric wake in which the asymmetric splitting process has disappeared and a more regular vortex street ensues. This result adds support to our hypothesis that the speed fluctuations play an important role in the splitting process and affect the ultimate structure and symmetry of the vortex wake.

In summary, we have suggested a physical explanation for why different vortex patterns arise in the wake of an oscillating cylinder. Using this explanation, we can construct a theoretical version of the WR map that closely tracks the experimental results by assuming that the region boundaries fall into two families of curves, one arising from the changes in direction of the velocity of the cylinder, the other from changes in its speed along its trajectory.

F.L.P. is indebted to the University of Buenos Aires for granting him a leave in order to complete this work. He would like to acknowledge the hospitality of the TAM Department at University of Illinois.

${ }^{1}$ O. M. Griffin and S. E. Ramberg, "The vortex street wakes of vibrating cylinders," J. Fluid Mech. 66, 553 (1974).

${ }^{2}$ A. Khalak and C. H. K. Williamson, "Motion forces and mode transitions in vortex-induced vibration at low mass-damping," J. Fluids Struct. 13, 813 (1999).

${ }^{3}$ C. H. K. Williamson and A. Roshko, "Vortex formation in the wake of an oscillating cylinder," J. Fluids Struct. 2, 355 (1988).

${ }^{4}$ F. Ponta and H. Aref, "The Strouhal-Reynolds number relationship for vortex streets," Phys. Rev. Lett. 93, 084501 (2004).

${ }^{5}$ R. Govardhan and C. H. K. Williamson, "Modes of vortex formation and frequency response of a freely vibrating cylinder," J. Fluid Mech. 420, 85 (2000).

${ }^{6}$ A. Roshko, "On the development of turbulent wakes from vortex streets," NACA TR 1191 (1954).

${ }^{7}$ C. H. K. Williamson and G. L. Brown, "A series in $1 / \sqrt{\text { Re }}$ to represent the Strouhal-Reynolds number relationship of the cylinder wake," J. Fluids Struct. 12, 1073 (1998). 